\title{
The feasibility of circulating tumor DNA analysis as a marker of recurrence in triple-negative breast cancer
}

\author{
SATOSHI OKAZAKI ${ }^{1,2}$, TAKAAKI SASAKI ${ }^{1}$, SHUNSUKE YASUDA $^{1,2}$, MASAHIRO ABE $^{1,2}$, NANA YOSHIDA $^{1,2}$, \\ RYOHEI YOSHIDA $^{1}$, KEI ISHIBASHI ${ }^{1,2}$, YOSHINORI MINAMI ${ }^{1}$, SHUNSUKE OKUMURA $^{1}$, SHINICHI CHIBA ${ }^{3}$, \\ HIDEHIRO TAKEI $^{4}$, RYUSUKE HAYASHI ${ }^{5,6}$, TOSHIHIRO NAGATO ${ }^{5,6}$, HIROYA KOBAYASHI $^{6}$, \\ AYUMU SUGITANI $^{7}$, YUSUKE ONO ${ }^{7}$, YUSUKE MIZUKAMI ${ }^{7,8}$, \\ MASAHIRO KITADA $^{1,2}$ and YOSHINOBU OHSAKI ${ }^{1}$ \\ ${ }^{1}$ Respiratory Center, ${ }^{2}$ Breast Center, ${ }^{3}$ Center for Advanced Research and Education, Asahikawa Medical University; \\ ${ }^{4}$ Department of Surgical Pathology, Asahikawa Medical University Hospital; \\ ${ }^{5}$ Department of Otolaryngology-Head and Neck Surgery; ${ }^{6}$ Department of Pathology, Asahikawa Medical University, \\ Asahikawa, Hokkaido 078-8510; ${ }^{7}$ Institute of Biomedical Research, Sapporo Higashi Tokushukai Hospital, \\ Sapporo, Hokkaido 065-0033; ${ }^{8}$ Division of Gastroenterology and Hematology/Oncology, \\ Department of Medicine, Asahikawa Medical University, Asahikawa, Hokkaido 078-8510, Japan
}

Received March 26, 2020; Accepted September 16, 2020

DOI: $10.3892 / 01.2021 .12681$

\begin{abstract}
Triple-negative breast cancer (TNBC) has a poorer prognosis than other breast cancer subtypes; therefore, identifying markers of early recurrence is important. The present study aimed to establish a liquid biopsy protocol for droplet digital PCR-based detection of frequently mutated genes in patients with TNBC. Tumor DNA from 36 patients with TNBC who relapsed within 2 years after surgical resection was retrospectively analyzed. Somatic mutational profiles were evaluated using targeted sequencing to identify frequently mutated genes and genes associated with molecularly targeted therapies. The association between genetic alterations and associated protein phosphorylation was investigated using immunohistochemical analysis. Recurrent hot spot mutations in the plasma were monitored over time. Mutation-specific probes were used to successfully detect mutations in the blood samples of patients who were positive for PIK3CA H1047R and AKT1 E17K mutations. Somatic mutations in AKT1 (14.9\%) and PIK3CA $(25.5 \%)$ were frequently identified in the data. Robust phosphorylation of AKT and S6RP was more common in tumors with PIK3CA H1047R and AKT1 E17K mutational background than in tumors with wild-type PIK3CA and AKT1. In conclusion, the present study evaluated a high-sensitivity detection system for frequently mutated genes that was also applicable for cell-free DNA. The PI3K/AKT pathway was
\end{abstract}

Correspondence to: Dr Takaaki Sasaki, Respiratory Center, Asahikawa Medical University, 2-1 Midorigaoka Higashi, Asahikawa, Hokkaido 078-8510, Japan

E-mail: takaaki6@asahikawa-med.ac.jp

Key words: triple-negative breast cancer, early recurrence, liquid biopsy, genetic testing, digital PCR revealed to be activated in patients harboring PIK3CA H1047R and $A K T 1$ E17K mutations; therefore, the PI3K/AKT pathway may be a promising candidate for targeted therapy in these patients.

\section{Introduction}

Triple-negative breast cancer (TNBC) accounts for $\sim 15 \%$ of all breast cancer cases and is defined by a lack of estrogen and progesterone receptors' expression and the absence of human epidermal growth factor receptor-2 (1). Molecular studies have demonstrated that TNBCs are a heterogeneous group of tumors with different clinical features, prognoses, genetic-molecular alterations, and responses to treatment (2). TNBCs are more likely to recur earlier after surgery than other subtypes, and many patients have poor prognosis (3). To increase success rate during TNBC treatment, personalized medicine designed for each patient's tumor profile individually taking genetic alterations in each patient into account is necessary.

Currently, for recurrence detection, serum tumor marker evaluation or computed tomography (CT) are used; however, more sensitive strategies should be developed to improve detection reliability. To identify relevant mutations, more accurate and cost-effective tools, such as strategies that use cell-free DNA (cfDNA), are required in clinical settings. However, the analysis of cfDNA using next-generation sequencing (NGS) is expensive and time-consuming. Among new technologies for cfDNA quantification, droplet digital PCR (ddPCR) provides the highest sensitivity for detecting and tracking actionable mutations at frequencies as low as $0.01-0.05 \%$ (4). In hormone receptor-positive breast cancer, the utility of liquid biopsy to analyze hormone therapy-resistant mutations has been studied $(5,6)$. In case of TNBC, liquid biopsy for detecting minimal residual disease during neoadjuvant chemotherapy was also studied and reported by some groups. These studies 
have demonstrated that circulating tumor DNA (ctDNA) level during neoadjuvant chemotherapy was associated with patient survival $(7,8)$. However, there is still no valid protocol that can be used for diagnosis of disease recurrence, which is focusing on specific mutations.

Here, we explore the practical utility of liquid biopsy using ddPCR-based detection of frequently mutated genes in samples of TNBC patients. We also describe the clinical course of metastatic TNBC patients whose genome harbors $A K T 1$ E17K and PIK3CA H1047R mutations, which have been reported to be oncogenic driver mutations in breast cancer that lead to PI3K/AKT pathway activation and tumor progression.

\section{Materials and methods}

Patients and breast cancer samples. We examined 57 consecutive TNBC patients who underwent mastectomy or breast-conserving surgery at the Asahikawa Medical University Hospital between April 2000 and March 2017. Thirty-six patients who relapsed within 2 years postoperatively were enrolled in the study. Tissue resected metastatic specimens of these patients were also evaluated. All cases were reviewed by two experienced pathologists and categorized according to the WHO classification (9). After surgical resection, patients underwent follow-up examination every 3 months for 2 years and every 6 months thereafter. The diagnosis of recurrence was made based on abnormal findings in the conserved mammary glands or by imaging diagnosis during the follow-up period. When patients had tumor recurrence, they were treated with chemotherapy (e.g., eribulin, tegafur-uracil, bevacizumab, paclitaxel, or vinorelbine).

This study protocol, including tumor sample collection and genetic analysis, was approved by the Asahikawa Medical University Research Ethics Committee (approval nos. 17043 and 18118). Written informed consent was obtained from all the patients before their enrollment for genetic analysis.

Sample collection and processing. Genomic DNA was extracted from formalin-fixed paraffin-embedded (FFPE) primary and recurrent tumor samples. Two cases were excluded because the specimens contained too few tumor cells. The tumor cell content ranged widely (from 20 to $90 \%$ ), with $44(94 \%)$ samples containing $>60 \%$ tumor cells. Viable tumor areas were dissected with a razor blade, and DNA was extracted using the GeneRead DNA FFPE Kit (Qiagen).

Plasma samples were collected from AKT1 E17K (c.49G>A)- and PIK3CA H1047R (c.3140A >G)-positive patients using PAXgene Blood ccfDNA Tube (Qiagen). Plasma was then isolated by centrifuging the samples at $1,400 \mathrm{xg}$ for $10 \mathrm{~min}$ at room temperature. The cell-free plasma was transferred into $2-\mathrm{ml}$ collection tubes and stored at $-80^{\circ} \mathrm{C}$ until purification. For cfDNA purification, QIAamp Circulating Nucleic Acid kit (Qiagen) was utilized. The genomic DNA concentration was measured using the Qubit dsDNA HS Assay kit (Thermo Fisher Scientific, Inc.) and the Qubit 3.0 fluorometer (Thermo Fisher Scientific, Inc.).

Targeted amplicon sequencing. To identify frequently mutated in our TNBCs and to determine which genetic alteration should be screening for cfDNA targeted amplicon sequencing was employed.

Between 20 and 100 nanograms of cfDNA were used for library construction with the Ion AmpliSeq Cancer Panel v2, which targets thousands of mutational hotspot regions in 50 cancer-associated genes using the Ion AmpliSeq Library Kit (Thermo Fisher Scientific, Inc.) (10). The sequencing was performed on an Ion PGM System according to the manufacturer's protocol, and sequencing leads were multiplexed, quality-filtered, and aligned to the human reference genome (GRCh37) using the Torrent Suite software (ver. 5.0.4; Thermo Fisher Scientific, Inc.). Variants were identified with the Variant Caller software (ver. 5.0.4.0; Thermo Fisher Scientific, Inc.) and filtered using Ion Reporter Software (Thermo Fisher Scientific, Inc.). The quality of all variants calls was manually confirmed by IGV software (ver. 2.3.59; Broad Institute, Cambridge, MA, USA).

Mutation detection by ddPCR. For the detection of the AKT1 c.49G $>$ A mutation, a specific primer/probe set was utilized (dHsaCP2000031; Bio-Rad Laboratories). For PIK3CA and TP53 mutations, specific assays were designed in-house and synthesized by Integrated DNA Technologies (IDT); nucleotide sequences of primers and probes are shown in Table SI). ddPCR assays were performed as previously described (11). Reaction mixtures were made of ddPCR Supermix for probes (no dUTP; Bio-Rad), primer, wild-type- and mutant-specific probes, and template DNA in a total volume of $22 \mu 1$. Droplets were generated by mixing the reaction mixture with Droplet Generation Oil (Bio-Rad Laboratories) in a QX200 droplet generator (Bio-Rad Laboratories). PCR was performed on a Veriti Thermal Cycler (Thermo Fisher Scientific, Inc.) using the following cycling conditions: $10 \mathrm{~min}$ at $95^{\circ} \mathrm{C}, 40$ cycles of $94^{\circ} \mathrm{C}$ for $30 \mathrm{sec}$ followed by $60^{\circ} \mathrm{C}$ for $T P 53$ or $57^{\circ} \mathrm{C}$ for PIK3CA for one minute, followed by $98^{\circ} \mathrm{C}$ for $10 \mathrm{~min}$. Samples were transferred to the QX200 Droplet Reader (Bio-Rad Laboratories) to measure the fluorescence of 6-fluorescein amidite and hexachloro-fluorescein probes. Droplets were scored as positive or negative based on their fluorescence intensity, which was determined by gating thresholds defined with positive and negative controls. Finally, absolute copy number input in the reaction and the ratio of mutated fragments were calculated by QuantaSoft ver 1.7 (Bio-Rad Laboratories) based on a Poisson distribution. Samples were scored as positive for the mutation when at least three mutation-containing droplets were detected by the mutant-specific probe as reported previously (12).

Immunohistochemical analysis. Immunohistochemistry (IHC) was performed using the Envision ${ }^{\mathrm{TM}}$ HRP System (\#K5361; Dako) using formalin-fixed sections, as described previously (13). Two samples with a low tumor cellularity were excluded. Heat-induced antigen retrieval was performed for phospho-AKT (pAKT; \#4060) and phospho-S6RP (pS6RP; \#4858; Cell Signaling Technology, Inc.) antibodies in EDTA buffer at $\mathrm{pH} 9.0$, and endogenous peroxidase activity was inhibited according to the manufacturer's instructions. The slides were then incubated at $4^{\circ} \mathrm{C}$ overnight with anti-pAKT at $1 / 25$ and anti-pS6RP at $1 / 100$ [diluted in Dako Real Antibody Diluent (Dako)], followed by incubation with an HRP-conjugated secondary antibody and substrate. 
Table I. Demographic and clinicopathological features of patients.

\begin{tabular}{|c|c|c|}
\hline Characteristics & $\mathrm{N}$ & $\%$ \\
\hline Total & 36 & \\
\hline Median age at surgery, years (range) & $56.6(30-81)$ & \\
\hline \multicolumn{3}{|l|}{ Menopausal status } \\
\hline Premenopausal & 13 & 36.1 \\
\hline Postmenopausal & 23 & 63.9 \\
\hline \multicolumn{3}{|l|}{ T stage } \\
\hline $\mathrm{T} 1(\leq 2.0 \mathrm{~cm})$ & 11 & 30.6 \\
\hline $\mathrm{T} 2(2.1-5.0 \mathrm{~cm})$ & 18 & 50 \\
\hline $\mathrm{T} 3(>5.0 \mathrm{~cm})$ & 5 & 13.9 \\
\hline $\begin{array}{l}\text { T4 (direct extension to the chest } \\
\text { wall or skin) }\end{array}$ & 2 & 5.5 \\
\hline \multicolumn{3}{|l|}{ No. of positive lymph nodes } \\
\hline 0 & 16 & 44.4 \\
\hline $1-3$ & 10 & 27.8 \\
\hline $4-9$ & 7 & 19.5 \\
\hline$\geq 10$ & 2 & 5.5 \\
\hline Unknown & 1 & 2.8 \\
\hline \multicolumn{3}{|l|}{ Stage } \\
\hline I & 7 & 19.5 \\
\hline II & 15 & 41.6 \\
\hline III & 10 & 27.8 \\
\hline IV & 4 & 11.1 \\
\hline \multicolumn{3}{|l|}{ Histological grade } \\
\hline 1 & 2 & 5.5 \\
\hline 2 & 7 & 19.5 \\
\hline 3 & 23 & 63.9 \\
\hline Unknown & 4 & 11.1 \\
\hline \multicolumn{3}{|l|}{ Histological type } \\
\hline Invasive ductal & 32 & 89 \\
\hline Invasive lobular & 2 & 5.5 \\
\hline Others & 2 & 5.5 \\
\hline \multicolumn{3}{|l|}{ Ki67 LI } \\
\hline$\leq 14$ & 2 & 5.5 \\
\hline$>14$ & 22 & 61.2 \\
\hline Unknown & 12 & 33.3 \\
\hline \multicolumn{3}{|l|}{ Chemotherapy received } \\
\hline Yes & 35 & 97.2 \\
\hline No & 1 & 2.8 \\
\hline Median PFS, months (range) & $12.6(0-24)$ & \\
\hline Median OS, months (range) & $31.9(1-175)$ & \\
\hline
\end{tabular}

LI, labeling index; PFS, progression-free survival; OS, overall survival.

Staining for pAKT and pS6RP was evaluated by consensus by two observers blinded to the clinical data. For scoring, the highest staining intensity in malignant cells was classified as 0 , no staining; 1 , weak staining; 2 , moderate staining; or 3, strong staining (Fig. S1).

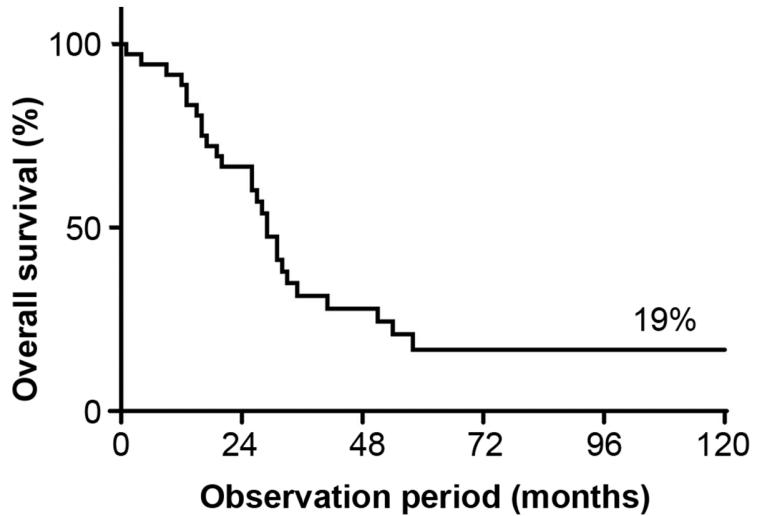

No. at risk

36

4

1

Figure 1. OS in TNBC patients who relapsed within 2 years after surgery. OS, overall survival; TNBC, triple-negative breast cancer.

Statistical analyses. Patients who were still alive at the most recent follow-up were censored for survival on the date of that follow-up. The Kaplan-Meier method was used to estimate overall survival (OS) rates. Fisher's exact test was used to compare our data on the frequency of gene mutations with that in the METABRIC database, which is the largest global study of breast cancer tissue samples ever performed (14). Pearson's correlation coefficient was calculated to examine the relevance of allele frequency obtained by targeted amplicon sequencing and ddPCR. The Mann-Whitney U test was used to assess IHC score differences between PIK3CA H1047R- and AKTI E17K-mutant samples and wild-type samples. A two-sided P-value of $<0.05$ was considered statistically significant. All statistical analyses were two-sided and performed using R software (version 3.4.1; The R Foundation, Vienna, Austria) (15).

\section{Results}

Characteristics of early recurrent TNBCs. The clinicopathological characteristics of the 36 enrolled patients are summarized in Table I. The 5-year OS rate was 19\% (Fig. 1). The median age at surgery was 56.6 years. Over half (63.9\%) of the patients were postmenopausal, and $89.0 \%$ had invasive ductal carcinoma histology. Four patients $(11.1 \%)$ were initially diagnosed with stage IV cancer. Neoadjuvant and adjuvant treatments were administered to 35 of 36 patients (97.2\%). Neoadjuvant chemotherapy was performed in 11 patients. Oral chemotherapy (tegafur-uracil) was administered to four patients. One patient did not receive chemotherapy because of liver cancer treatment. The median time to progression and median OS from surgery were 12.6 months (range: 0-24 months) and 31.9 months (range: 1-175 months), respectively.

Genetic alterations in early recurrent TNBCs. Of the 36 cases, the tumor content was low in two cases, so they were excluded from DNA analysis. There were 13 patients who had undergone resection of the recurrence, each with eight lymph nodes, two lungs, two brains, and one pleura. Of 1,835 variants obtained from 47 samples, 72 variants were selected by coverage, type of function, sequencing quality, filtering of single-nucleotide 


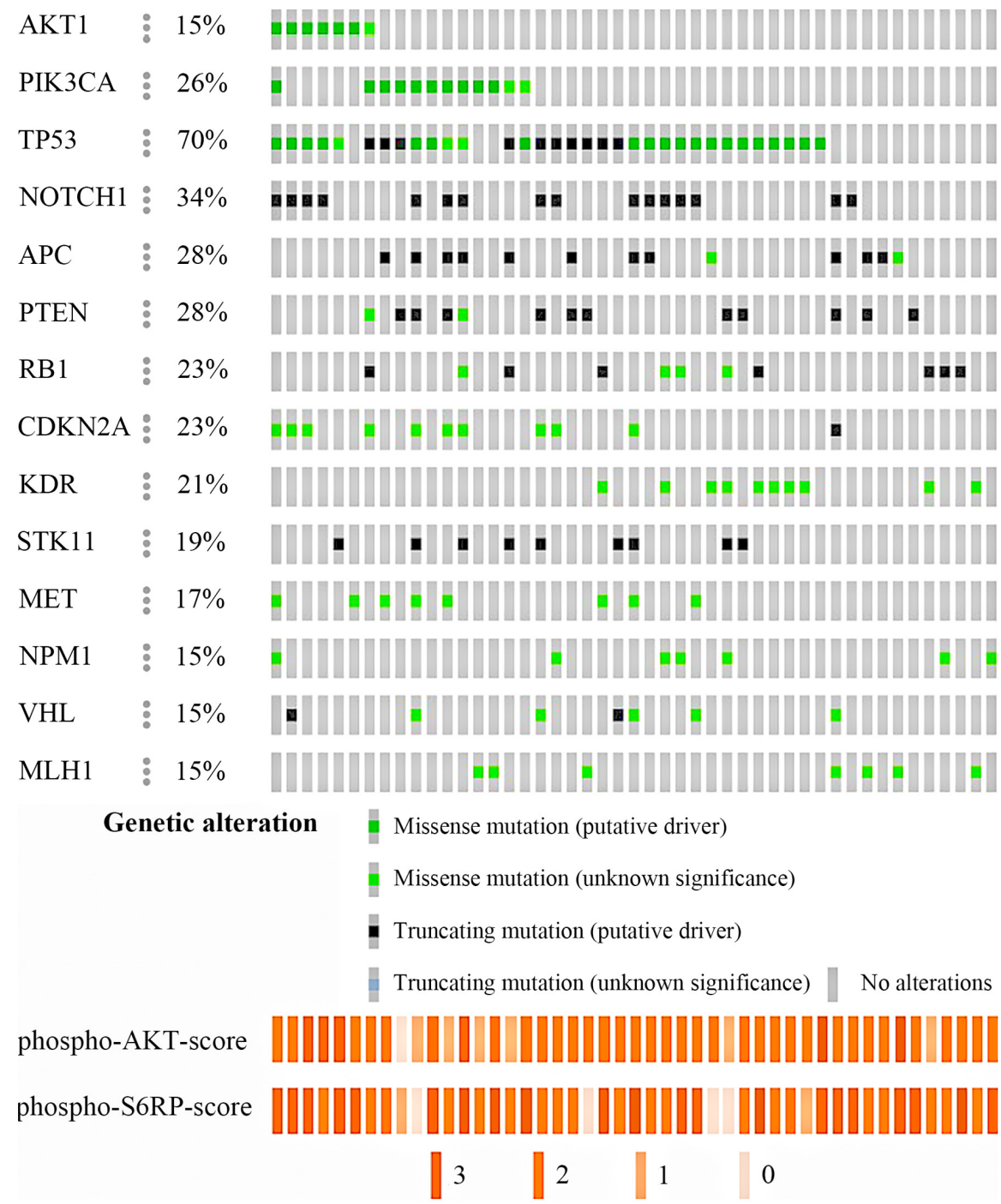

Figure 2. Mutation frequencies and IHC score in 47 TNBC samples. DNA was extracted from 34 primary and 13 recurrent tumors and IHC was performed on 47 similar samples. Mutation analysis of cancer-related genes that were mutated in at least $15 \%$ of 47 TNBC samples show phospho-AKT (upper) and phospho-S6RP (lower) IHC scores of the samples. IHC, immunohistochemistry; TNBC, triple-negative breast cancer; RP, ribosomal protein.

polymorphisms (SNPs) using Variant Caller software, and manual selection of false-positives (Fig. S2) (16). Forty-eight were single nucleotide variants $(66.7 \%)$ and 24 were small insertion-deletions (33.3\%). Of these variants, 54 and seven were unique to the primary and metastatic lesions, respectively, and 11 were found in both. Thirty-three of 34 tumors (97.1\%) obtained from primary tumors had the following mutations (average 5.2, range 1-12 mutations): TP53 (27, 79.4\%); NOTCH1 (16, 47.1\%); PTEN (13, 38.2\%); PIK3CA (11, 32.3\%); CDKN2A (11, 32.3\%); APC (10, 29.4\%); VHL (8, 23.5\%); and AKT1 (7, 20.6\%) (Fig. 2). All 13 recurrent samples harbored mutations (average 2.5, range: 1-5 mutations), and mutation in $K D R$ was the most frequent $(8,61.5 \%)$. The comparison of genetic mutations in 13 metastatic tumors and corresponding primary tumors were shown in Fig. S3. The left side column shows the data of the primary tumor and the right column shows the data of the metastatic tumor. $A K T 1$ mutations were not found in these samples. For PIK3CA mutation, samples from 4 patients were detected including from 3 primary tumors. The sites and frequencies of mutations in TP53, AKT1, and PIK3CA are shown in Fig. 3. AKT1 E17K was the most common mutation in our study (7/47, 14.9\%) (Fig. 3A). For PIK3CA gene (Fig. 3B), PIK3CA H1047R was the most frequent mutation $(5 / 47,10.6 \%)$. In contrast, for mutations in TP53 gene, there were various mutation sites, but no characteristic mutations were found (Fig. 3C).

Patterns of expression of phospho-AKT and phospho-S6RP. $A K T 1$ and $P I K 3 C A$ were reported as driver oncogenes whose activity was sufficient to transform a normal cell into a cancerous one. PI3K/AKT signaling pathway activity can be detected by the phosphorylation of AKT and S6RP proteins. Therefore, we investigated the correlation between genetic alterations and protein phosphorylation in tumor samples by IHC (Fig. 2). Representative images of tumor cells with strong, moderate, weak, and no staining for pAKT1 and pS6RP are shown in Fig. S1. Tumors with PIK3CA H1047R or AKT1 E17K mutations had higher expression level of phospho-AKT (pAKT) and phospho-S6RP (pS6RP) than PIK3CA and $A K T 1$ wild-type tumors $(\mathrm{P}=0.044$ and $\mathrm{P}=0.105$, respectively; Table SII), suggesting that genetic alteration of $A K T 1$ or PIK3CA leads to induction of abnormal activation of survival pathways in cancer. 


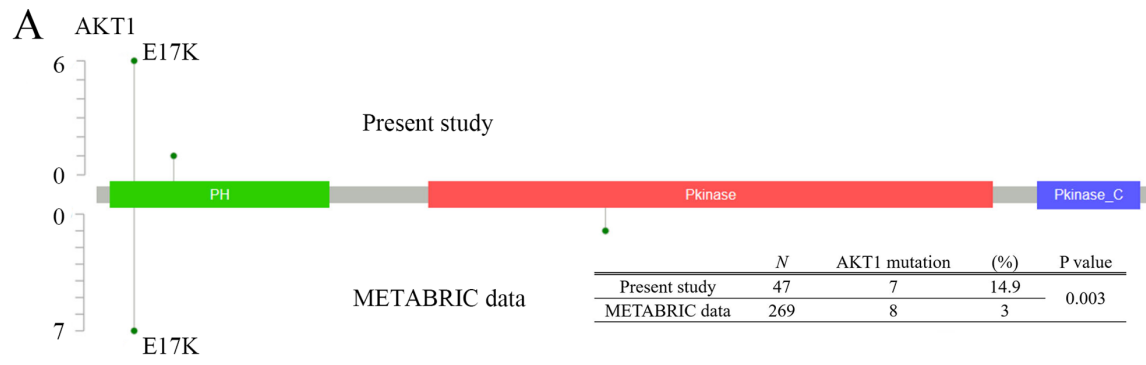

B PIK3СA

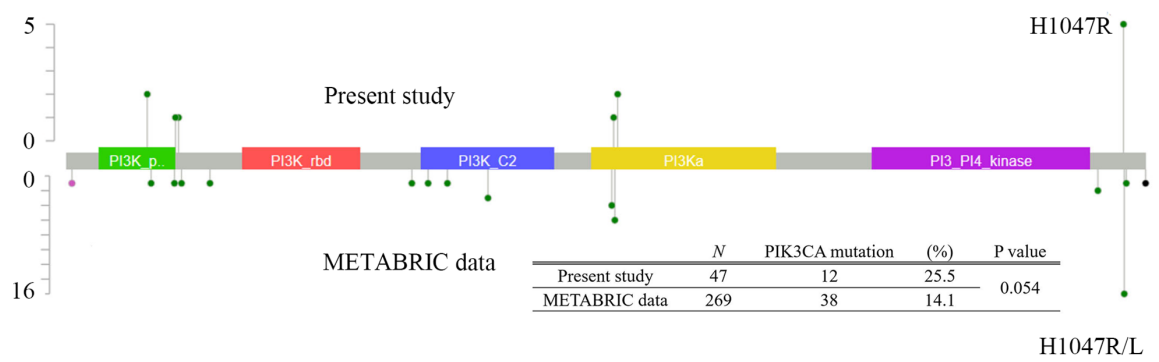

C TP53

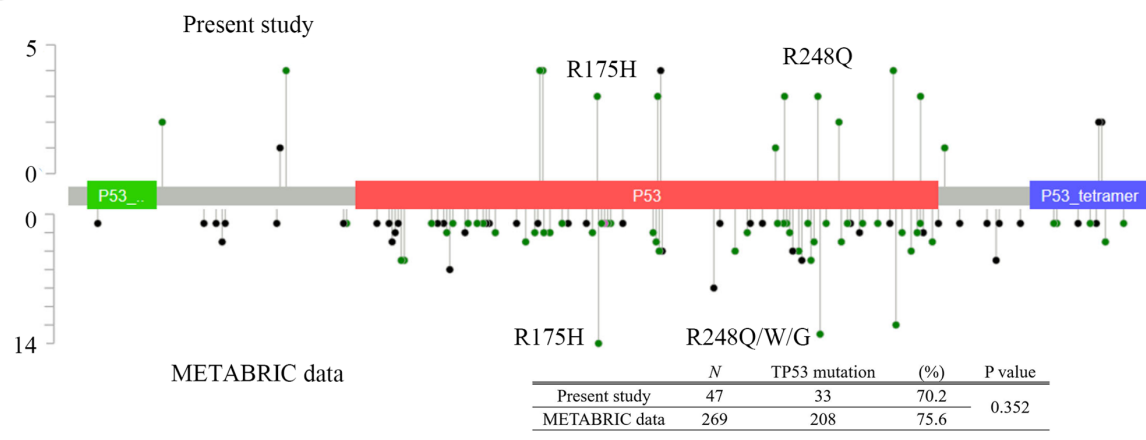

Figure 3. Sites distribution of (A) AKT, (B) PIK3CA and (C) TP53 mutation. The table at the bottom shows a comparison between mutations detected in our data (upper line) and in the METABRIC cohort (lower line). P-values were calculated using Fisher's exact test.

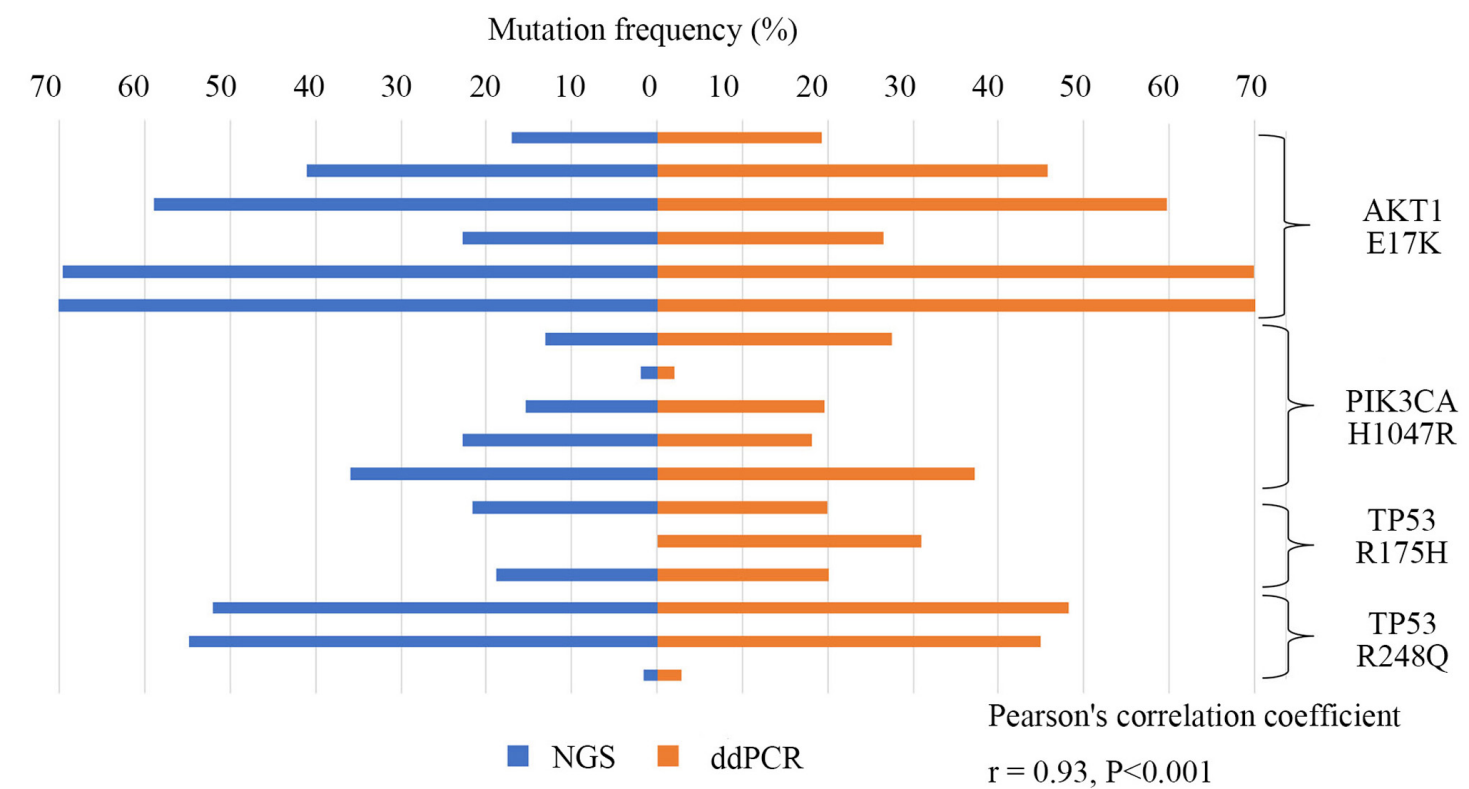

Figure 4. Specific mutation frequencies of TNBC as detected by NGS and ddPCR. We designed or purchased allele-specific probes for ddPCR. We then used these probes to validate the mutation (AKT1 E17K, PIK3CA H1047R, and TP53 R175H and R248Q) allele frequencies in NGS and ddPCR data of the DNA derived from 47 specimens of early recurrent TNBC. P-values and R were calculated using Pearson's correlation coefficient. TNBC, triple-negative breast cancer; NGS, next-generation sequencing; ddPCR, droplet digital polymerase chain reaction. 
A Patient no. 2443
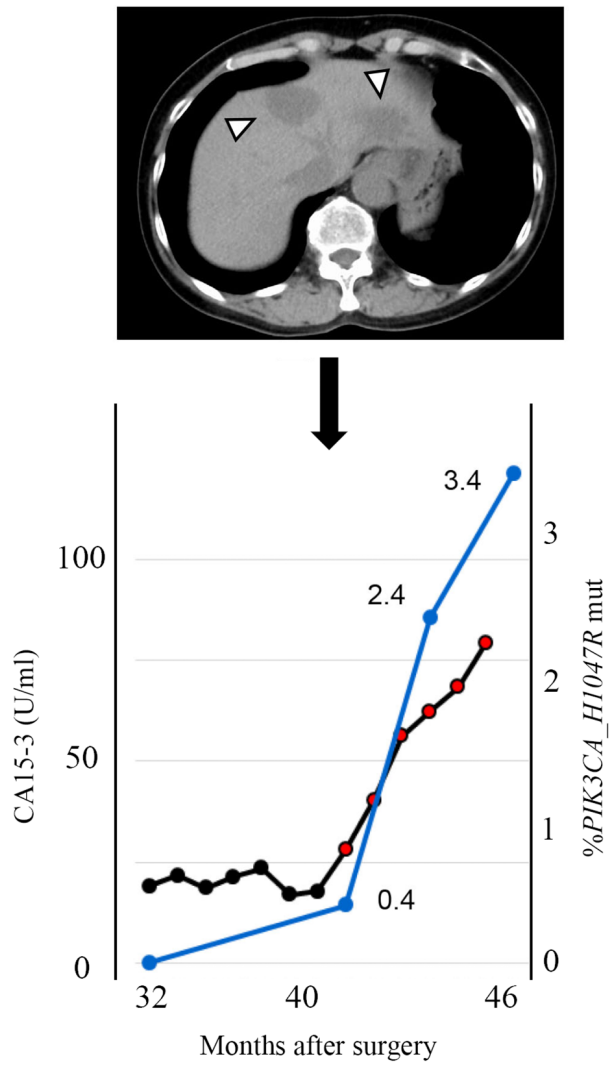

CA15-3 $\%$
B

Patient no. 2152

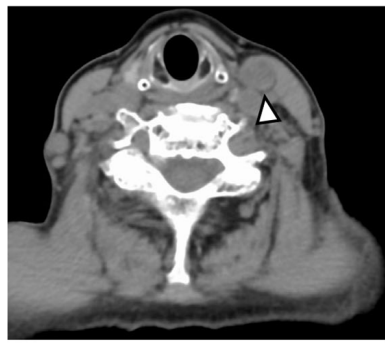

10
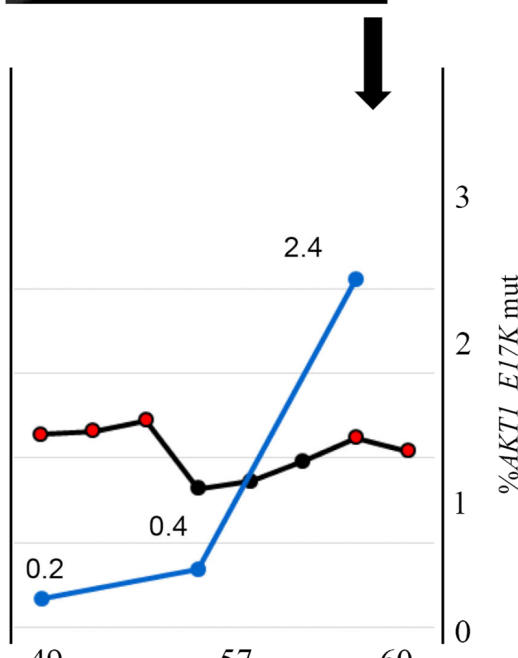

49

57

60

Months after surgery

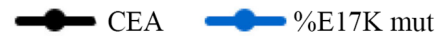

Figure 5. Clinical course of case reports. (A) A 58-year-old woman with a diagnosis of TNBC. The graph shows the change in the percentage of PIK3CA H1047R mutations detected in cfDNA using ddPCR and the CA15-3 levels during the clinical course after surgery. CT shows multiple liver metastases 13 months after surgery and also the progression of liver metastases 25 months after surgery. CT shows further progression of the liver metastases 41 months after surgery. Arrowhead indicates liver metastasis. (B) A 77-year-old woman with Stage IIIB TNBC with direct extension to the skin and lymph node metastasis. The graph shows the change in the percent E17K mutation detected in cfDNA using ddPCR and the CEA levels during the clinical course after surgery. CT shows lymph node progression 11 months after surgery and 48 months after surgery. CT shows new left cervical lymph node metastasis. Arrowhead indicates lymph node metastasis. cfDNA, cell-free DNA; ddPCR, droplet digital polymerase chain reaction; CT, computed tomography; CA15-3, carbohydrate antigen 15-3; CEA, carcinoembryonic antigen.

Mutation allele frequencies of FFPE samples. We sought to develop a highly sensitive blood-based method for detection of genetic alterations that can be used as a clinical application for serial monitoring. We designed or purchased allele-specific probes for ddPCR that yielded $0.01 \%$ detection sensitivity between mutant and wild-type alleles (Table SI; Fig. S4; Appendix S1). Genomic DNA extracted from HCT116, SKBR-3 or HCC70 cells was used as a ddPCR mutation control for PIK3CA H1047R, TP53 R175H or TP53 R248Q, respectively, and genomic DNA extracted from HCC 38 cells was used as a wild-type control for PIK3CA or TP53 (Table SIII).

Then the frequencies of mutations identified by ddPCR were compared to the ones previously determined by NGS using DNA derived from the FFPE tissue samples containing 47 specimens of early TNBC recurrence. As shown in Fig. 4, AKT1 E17K, PIK3CA H1047R, and TP53 R175H and R248Q mutations detected by NGS significantly correlated with frequencies in ddPCR $(r=0.93, \mathrm{P}<0.001)$.

Results of digital PCR detection of clinical samples. In our early recurrent TNBCs patients, 4 patients out of 36 patients still survived. Among them, consent was obtained in three cases, and plasma was collected and analyzed. Patients nos. 1422 and 2443 were harboring PIK3CA H1047R mutation and patient no. 2152 was harboring $A K T 1$ E17K mutation in primary tumor, however in patient no. 1422, PIK3CA H1047R mutation was not detected in lymph node metastasis site nor cfDNA due to the adjuvant chemotherapy after surgery (Fig. S5A).

In other TNBC cohort including 3 cases with early recurrence within 2 years after surgery, 2 cases of late recurrence, 4 cases without metastasis were analyzed for cfDNA. Within this cohort, 2 patients (patient nos. 2667 and 3019) were harboring PIK3CA H1047R (Fig. S5B and C) or a patient (patient no. 996) was harboring AKT1 E17K mutation (Fig. S5D) and all corresponding cfDNA from plasma was detected in the same mutations as primary lesion.

Collectively, 6 patients with metastatic TNBC patients harboring PIK3CA H1047R or AKT1 E17K mutation, 5 patients $(83.3 \%)$ were detected corresponding mutation by digital PCR methods.

Clinical course of a PIK3CA H1047R-positive TNBC patient. A 58-year-old woman (patient no. 2443) with a diagnosis of 
TNBC (T3N0M0, Stage IIb) received neoadjuvant chemotherapy with 5-fluorouracil/epirubicin/cyclophosphamide followed by docetaxel. After neoadjuvant chemotherapy, she underwent mastectomy and sentinel lymph node biopsy. Histological examination of the resected specimens revealed pathological complete response. Needle biopsy revealed invasive lobular carcinoma with high proliferative rate (MIB-1 index 28\%). Thirteen months after the surgery, multiple liver metastases were found. Furthermore, 39 months after surgery, a liquid biopsy was performed (and the cfDNA was extracted, concentration: $20.6 \mathrm{ng} / \mathrm{ml}$ of plasma). Genetic mutation analysis of cfDNA indicated the presence of PIK3CA c. $3140 \mathrm{~A}>\mathrm{G}$ mutation (H1047R), as did genetic analysis of the primary site, and the allele frequency was $0.4 \%$ as defined by the liquid biopsy. One month later, progression of liver metastases was detected by CT (Fig. 5A). Liquid biopsies were performed every three months thereafter, and the increase of mutant allele frequency was correlated to increase of CA-15-3. As shown in Fig. S5B and C, mutations were detectable by liquid biopsy in two other patients, and one of them had brain metastasis while a tumor marker was negative.

Clinical course of an AKT1 E17K-positive TNBC patient. A 77-year-old woman (patient no. 2152) underwent left breast resection and axillary lymph node dissection. She was diagnosed with Stage IIIB TNBC with direct extension to the skin and lymph node metastasis. Examination of the biopsy sample revealed invasive ductal carcinoma with high proliferative rate (MIB-1 index 38\%). Eleven months after surgery, CT confirmed lymph node progression. Fifty-two months after surgery, a liquid biopsy was performed and the concentration of extracted cfDNA was $16.6 \mathrm{ng} / \mathrm{ml}$ of plasma. Seven months later, a second liquid biopsy was performed and the concentration of extracted cfDNA was $18.8 \mathrm{ng} / \mathrm{ml}$ of plasma. Ten months later, a third liquid biopsy was performed and the concentration of cfDNA was $13.5 \mathrm{ng} / \mathrm{ml}$ of plasma. Genetic mutation analysis of cfDNA indicated the presence of AKT1 c. $49 \mathrm{G}>\mathrm{A}(\mathrm{E} 17 \mathrm{~K})$ as in the primary site, and the mutation allele frequency was $0.2 \%$ in the first biopsy and $0.4 \%$ in the second biopsy (Fig. 5B). In the second liquid biopsy, although tumor markers were undetectable, the AKT1 c.49G >A (E17K) mutation could be detected. In the third liquid biopsy, the allele frequency rose to $2.4 \%$, and one month later, metastasis of left the cervical lymph node was detected by CT. As shown in Fig. S5D, mutation was detectable by liquid biopsy in another patient.

\section{Discussion}

We studied a high-sensitivity detection system based on ddPCR that enabled the detection of low-level copy mutations and the identification of mutations in plasma samples of patients with metastatic TNBC. ddPCR is a relatively inexpensive method and has rapid turnover. The ddPCR assay is especially useful when there is insufficient genomic DNA for NGS analysis. Circulating DNA in the cell-free plasma fraction originates from many different cells, including lymphocytes and neoplastic cells (17). Their DNA is released into the circulation in the process of cellular destruction (18).
Therefore, circulating-tumor DNA (ctDNA) in cell-free plasma represents a very low fraction of the total amount of circulating DNA, but there are several reports in which ctDNA in TNBC patients was analyzed. Chen et al (7) reported that ctDNA was detected in four out of the 33 early-stage TNBC patients after neoadjuvant chemotherapy by NGS analysis. All four cases relapsed early after surgery, and disease-free survival (DFS) was significantly inferior compared to cases in which ctDNA could not be detected. On the other hand, ctDNA could not be detected in nine of the recurrent cases, and the sensitivity remained at $31 \%$. Riva et al (8) reported that in 27 out of 36 early TNBC patients before treatment, it was possible to detect ctDNA, and in patients, for whom it was possible to detect ctDNA even after 1 cycle of neoadjuvant chemotherapy, DFS and OS were significantly lower. These findings suggest that the detection and quantification of ctDNA is a very promising tool for assessing a response to neoadjuvant chemotherapy. Our detection system using ddPCR can be used for liquid biopsy because of its high sensitivity. Importantly, using this protocol, we were able to understand the disease progression during chemotherapy in patients with metastatic TNBC. In patient no. 2152 in particular, an increase in the mutation copy ratio was observed reflecting on the disease state progression more sensitively than the tumor marker did. In solid tumors other than breast cancer, biomarkers have been used as a tool to detect recurrence. These biomarkers include prostate-specific antigen for prostate cancer, carcinoembryonic antigen (CEA) for colon cancer, and cancer antigen 125 for ovarian cancer (19). On the other hand, in breast cancer or non-small cell lung cancer, Clinical Practice Guidelines does not recommend the surveillance tumor marker testing for the patients treated with curative intent. It because biomarkers measured postoperatively were not sensitive nor specific for cancer relapse $(20,21)$.

In our previous study, the usefulness of cfDNA measurements rather than tumor markers in NSCLC patients was indicated (11). Ideally, if we could design digital PCR probes/primers for all the detected genetic mutations by NGS, all patients could be eligible for detection from cfDNA. However, NGS was not performed for the purpose of screening of functioning genetic mutations in TNBCs, but rather for genetic mutations that should be searched in cfDNA. In the present study, we identified frequently mutated and associated with molecularly targeted therapies using NGS based genetic alteration screening and we focused on AKT1 and PIK3CA mutations and showed that analysis of cfDNA over time could be useful for determining recurrence.

The prognosis is poor for metastatic TNBC and TNBC that recurs within 2-3 years (1). Although clinical trials have examined various treatments (22-24), no cure has been established. In this study, we found that PI3K/AKT signaling, and in particular PIK3CA H1047R or AKT1 E17K mutations, were significantly associated with high incidence of metastasis as compared to the METABRIC cohort. The METABRIC database contains the details of genetic mutations for 269 TNBC cases, which we compared to our results (Fig. 3). The frequency of $A K T 1$ mutation was higher in our study than in METABRIC. AKT1 E17K was the most common mutation in both groups (Fig. 3A). For PIK3CA gene, the mutation sites were generally consistent between 
our data and those in METABRIC; however, the mutational frequency was not statistically significant, although there was a tendency for higher mutational frequency in our study compared with that shown in METABRIC (Fig. 3B). In both groups, PIK3CA H1047R was the most frequent mutation (our data: 5/47, 10.6\% and METABRIC: 15/269, 5.6\%). While Takeshita et al (5) reported that the presence of PIK3CA H1047R or E542K in cfDNA was associated with favorable prognosis, in our cohort, patients with recurrence within two years after surgery were corrected in this study, therefore we could not verify whether PIK3CA H1047R was prognosis marker or not. In contrast, the mutations in TP53 were generally consistent, but no statistically significant difference in its frequency was observed (Fig. 3C). The frequencies of TP53 R175H and R248Q mutations were higher in METABRIC (13/269, 4.8\% and 7/269, 2.6\%, respectively), but these mutations were also common in our study $(3 / 47,6.4 \%)$. A previous report suggested that Asian TNBC patients were more likely to harbor PI3K/AKT mutations than patients of other ethnicities (25). AKT1 E17K is considered a driver oncogene (26-28), and AKT inhibitors have been assessed in preclinical and clinical trials in multiple cancer types known to harbor this mutation, suggesting that AKT-targeting therapy may be effective for patients with activated AKT signaling (29). Based on the above, Asian TNBC patients have a higher frequency of this mutation, and AKT inhibitors may be an effective treatment option for this population.

There are limitations in this study. First, the number of patient samples was small; however, we analyzed 36 patients with TNBC who relapsed within 2 years after surgical resection, the frequency of $A K T 1 \mathrm{E} 17 \mathrm{~K}$ was higher in this cohort, and there may be clinical benefits in monitoring this mutation, as it is a marker of recurrence. A long-term surveillance study in a large cohort of patients is warranted to confirm these important findings. Second, the liquid biopsy was performed on patients whose tumor has already metastasized. For early detection, it is necessary to confirm similar events in patients whose tumor has not yet metastasized.

Nevertheless, we believe that molecular target therapy for druggable driver oncogene will be applicable for the treatment of TNBC in the near future. Intervention with kinase inhibitors could improve prognosis as seen in other types of cancers. So, it is important to know what kinds of driver oncogene are present in TNBC patients with poor prognosis that can be detected in cfDNA mutation analysis by cost-effective and highly sensitive way.

In this study, considering the possibility for AKT inhibitor treatment as a therapy for TNBC, liquid biopsy was performed on patients with $A K T 1$ and PIK3CA mutations. Based on the results obtained, detecting single mutation detection like PIK3CA and AKT for surveillance of TNBC which comprised $\sim 32 \%$ of TNBC was not sufficient for the early detection of TNBC recurrence; however, we believe that using predefined genetic alteration, multiple targets in ddPCR, e.g., TP53 R175H/R248Q or high-sensitive NGS-based liquid biopsy could be employed to yield higher detection rates.

In conclusion, in our TNBC early recurrent patient cohort, AKT1 E17K (14.9\%) and PIK3CA H1047R (10.6\%) were common in surgically resected samples detected by target amplicon sequencing. The PI3K/AKT pathway was active in patients harboring $A K T 1$ E17K and PIK3CA H1047R mutations, suggesting an inhibitor of this pathway needed to be explored as a possible therapeutic agent. Finally, we successfully detected predefined genetic mutations from circulating free DNA using ddPCR method; however, for early recurrence detection or treatment monitoring, we suggest using multiple target detection strategy with high detection sensitivity.

\section{Acknowledgements}

The authors would like to thank Dr Munehiko Ogata and Ms. Mayumi Suzuki (Sapporo Higashi Tokushukai Hospital) for technical support during genetic analyses.

\section{Funding}

This work was supported by JSPS KAKENHI [grant no. $18 \mathrm{~K} 15262$ (to RY)]

\section{Availability of data and materials}

The datasets generated and/or analyzed during the current study are not publicly available due to their containing genetic information that could compromise the privacy of research participants but are available from the corresponding author on reasonable request.

\section{Authors' contributions}

Conception and design: $\mathrm{SaO}$ and TS. Provision of study materials, and acquisition, analysis and interpretation of data: SaO, TS, SY, MA, NY, RY, KI, YoM, ShO, SC, HT, RH, TN, HK, AS, YOn, YuM, MK and YOh. All authors wrote, read and approved the final manuscript.

\section{Ethics approval and consent to participate}

This study protocol, including tumor sample collection and genetic analysis, was approved by the Asahikawa Medical University Research Ethics Committee (approval nos. 17043 and 18118). For the prospective study (approval nos. 17043), written informed consent was obtained from all patients before their enrollment for genetic analysis. For the retrospective study for survival analysis of 36 patients with TNBC (approval no. 18118), informed consent was waived by the Asahikawa Medical University Research Ethics Committee and an opportunity for opt-out regarding the study was provided through the institutional website.

\section{Patient consent for publication}

Written informed consent for publication of clinical details and /or clinical images was obtained from the patients.

\section{Competing interests}

YMiz and YOn receive funding from Hitachi High Technologies, Inc. for the experimental equipment. No potential conflicts of interest were disclosed by the other authors. 


\section{References}

1. Massihnia D, Galvano A, Fanale D, Perez A, Castiglia M, Incorvaia L, Listì A, Rizzo S, Cicero G, Bazan V, et al: Triple negative breast cancer: Shedding light onto the role of pi3k/akt/mtor pathway. Oncotarget 7: 60712-60722, 2016.

2. Cossu-Rocca P, Orrù S, Muroni MR, Sanges F, Sotgiu G, Ena S, Pira G, Murgia L, Manca A, Uras MG, et al: Analysis of PIK3CA mutations and activation pathways in triple negative breast cancer. PLoS One 10: e0141763, 2015.

3. Dent R, Trudeau M, Pritchard KI, Hanna WM, Kahn HK, Sawka CA, Lickley LA, Rawlinson E, Sun P and Narod SA: Triple-negative breast cancer: Clinical features and patterns of recurrence. Clin Cancer Res 13: 4429-4434, 2007.

4. Oxnard GR, Paweletz CP, Kuang Y, Mach SL, O'Connell A, Messineo MM, Luke JJ, Butaney M, Kirschmeier P, Jackman DM, et al: Noninvasive detection of response and resistance in EGFR-mutant lung cancer using quantitative next-generation genotyping of cell-free plasma DNA. Clin Cancer Res 20: 1698-1705, 2014.

5. Takeshita T, Yamamoto Y, Yamamoto-Ibusuki M, Tomiguchi M, Sueta A, Murakami K and Iwase H: Clinical significance of plasma cell-free DNA mutations in PIK3CA, AKT1, and ESR1 gene according to treatment lines in ER-positive breast cancer. Mol Cancer 17: 67, 2018.

6. Takeshita T, Yamamoto Y, Yamamoto-Ibusuki M, Tomiguchi M, Sueta A, Murakami K, Omoto Y and Iwase H: Analysis of ESR1 and PIK3CA mutations in plasma cell-free DNA from ER-positive breast cancer patients. Oncotarget 8: 52142-52155, 2017.

7. Chen YH, Hancock BA, Solzak JP, Brinza D, Scafe C, Miller KD and Radovich M: Next-generation sequencing of circulating tumor DNA to predict recurrence in triple-negative breast cancer patients with residual disease after neoadjuvant chemotherapy. NPJ Breast Cancer 3: 24, 2017.

8. Riva F, Bidard FC, Houy A, Saliou A, Madic J, Rampanou A, Hego C, Milder M, Cottu P, Sablin MP, et al: Patient-Specific Circulating Tumor DNA Detection during Neoadjuvant Chemotherapy in Triple-Negative Breast Cancer. Clin Chem 63: 691-699, 2017

9. Lakhani SR, Ellis IO and Schnitt SJ, Tan $\mathrm{pH}$ and van de Vijver MJ (eds): WHO Classification of Tumours of the Breast. Vol 4. 4th edition. IARC WHO Classification of Tumours, 2012

10. Yoshida R, Sasaki T and Ohsaki Y: EGFR and KRAS mutations in triple-mutated lung cancer. J Thorac Oncol 12: e92-e93, 2017.

11. Yoshida R, Sasaki T, Umekage Y, Tanno S, Ono Y, Ogata M, Chiba S, Mizukami Y and Ohsaki Y: Highly sensitive detection of ALK resistance mutations in plasma using droplet digital PCR. BMC Cancer 18: 1136, 2018.

12. Ono Y, Sugitani A, Karasaki H, Ogata M, Nozaki R, Sasajima J, Yokochi T, Asahara S, Koizumi K, Ando K, et al: An improved digital polymerase chain reaction protocol to capture low-copy KRAS mutations in plasma cell-free DNA by resolving 'subsampling' issues. Mol Oncol 11: 1448-1458, 2017.

13. Ishibashi K, Kumai T, Ohkuri T, Kosaka A, Nagato T, Hirata Y, Ohara K, Oikawa K, Aoki N, Akiyama N, et al: Epigenetic modification augments the immunogenicity of human leukocyte antigen $\mathrm{G}$ serving as a tumor antigen for $\mathrm{T}$ cell-based immunotherapy. OncoImmunology 5: e1169356, 2016.

14. Pereira B, Chin SF, Rueda OM, Vollan HK, Provenzano E, Bardwell HA, Pugh M, Jones L, Russell R, Sammut SJ, et al: The somatic mutation profiles of 2,433 breast cancers refines their genomic and transcriptomic landscapes. Nat Commun 7: 11479, 2016.
15. Kanda Y: Investigation of the freely available easy-to-use software 'EZR' for medical statistics. Bone Marrow Transplant 48: 452-458, 2013

16. McCall CM, Mosier S, Thiess M, Debeljak M, Pallavajjala A, Beierl K, Deak KL, Datto MB, Gocke CD, Lin MT, et al: False positives in multiplex PCR-based next-generation sequencing have unique signatures. J Mol Diagn 16: 541-549, 2014.

17. Schwarzenbach H, Hoon DS and Pantel K: Cell-free nucleic acids as biomarkers in cancer patients. Nat Rev Cancer 11: 426-437, 2011.

18. Bettegowda C, Sausen M, Leary RJ, Kinde I, Wang Y, Agrawal N, Bartlett BR, Wang H, Luber B, Alani RM, et al: Detection of circulating tumor DNA in early- and late-stage human malignancies. Sci Transl Med 6: 224ra24, 2014

19. Barak V, Holdenrieder S, Nisman B and Stieber P: Relevance of circulating biomarkers for the therapy monitoring and follow-up investigations in patients with non-small cell lung cancer. Cancer Biomark 6: 191-196, 2010.

20. Colt HG, Murgu SD, Korst RJ, Slatore CG, Unger M and Quadrelli S: Follow-up and surveillance of the patient with lung cancer after curative-intent therapy: Diagnosis and Management of Lung Cancer, 3rd ed: American College of Chest Physicians Evidence-Based Clinical Practice Guidelines. Chest 143 (Suppl 5): e437S-e454S, 2013.

21. Emens LA and Davidson NE: The follow-up of breast cancer. Semin Oncol 30: 338-348, 2003.

22. Albain KS, Nag SM, Calderillo-Ruiz G, Jordaan JP, Llombart AC, Pluzanska A, Rolski J, Melemed AS, Reyes-Vidal JM, Sekhon JS, et al: Gemcitabine plus Paclitaxel versus Paclitaxel monotherapy in patients with metastatic breast cancer and prior anthracycline treatment. J Clin Oncol 26: 3950-3957, 2008.

23. Perez EA, Hillman DW, Mailliard JA, Ingle JN, Ryan JM, Fitch TR, Rowland KM, Kardinal CG, Krook JE, Kugler JW, et al: Randomized phase II study of two irinotecan schedules for patients with metastatic breast cancer refractory to an anthracycline, a taxane, or both. J Clin Oncol 22: 2849-2855, 2004.

24. Talbot DC, Moiseyenko V, Van Belle S, O'Reilly SM, Alba Conejo E, Ackland S, Eisenberg P, Melnychuk D, Pienkowski T, Burger HU, et al: Randomised, phase II trial comparing oral capecitabine (Xeloda) with paclitaxel in patients with metastatic/advanced breast cancer pretreated with anthracyclines. Br J Cancer 86: 1367-1372, 2002.

25. Jiang YZ, Ma D, Suo C, Shi J, Xue M, Hu X, Xiao Y, Yu KD, Liu YR, Yu Y, et al: Genomic and transcriptomic landscape of triple-negative breast cancers: Subtypes and treatment strategies. Cancer Cell 35: 428-440.e5, 2019.

26. de Bruin EC, Whiteley JL, Corcoran C, Kirk PM, Fox JC, Armisen J, Lindemann JPO, Schiavon G, Ambrose HJ and Kohlmann A: Accurate detection of low prevalence AKT1 E17K mutation in tissue or plasma from advanced cancer patients. PLoS One 12: e0175779, 2017.

27. Kim MS, Jeong EG, Yoo NJ and Lee SH: Mutational analysis of oncogenic AKT E17K mutation in common solid cancers and acute leukaemias. Br J Cancer 98: 1533-1535, 2008.

28. Rudolph M, Anzeneder T, Schulz A, Beckmann G, Byrne AT, Jeffers M, Pena C, Politz O, Köchert K, Vonk R, et al: AKT1 $(\mathrm{E} 17 \mathrm{~K})$ mutation profiling in breast cancer: Prevalence, concurrent oncogenic alterations, and blood-based detection. BMC Cancer 16: 622, 2016.

29. Hyman DM, Smyth LM, Donoghue MTA, Westin SN, Bedard PL, Dean EJ, Bando H, El-Khoueiry AB, Pérez-Fidalgo JA, Mita A, et al: AKT inhibition in solid tumors with AKT1 mutations. J Clin Oncol 35: 2251-2259, 2017. 